\title{
Moxetumomab Pasudotox
}

National Cancer Institute

\section{Source}

National Cancer Institute. Moxetumomab Pasudotox. NCI Thesaurus. Code C68819.

A recombinant immunotoxin consisting of the Fv portion of the anti-CD22 antibody covalently fused to a $38 \mathrm{KD}$ a fragment of Pseudomonas exotoxin-A (PE38) with potential antineoplastic activity. The Fv portion of anti-CD22 immunotoxin CAT-8015 binds to CD22, a cell surface receptor expressed on a variety of malignant B-cells, thereby delivering the toxin moiety PE38 directly to tumor cells. Once internalized, PE38 induces caspase-mediated apoptosis via a mechanism involving mitochondrial damage and blocks translational elong ation by binding to elong ation factor 2 (EF-2). Anti-CD22 immunotoxin CAT -8015 exhibits a greater affinity for CD22 than its predecessor, antiCD22 immunotoxin CAT-3888 (BL22 immunotoxin), and hence may be more effective against tumor cells expressing lower levels of CD22. 